\title{
Environmental contours for mixtures of distributions
}

\author{
Arne Bang Huseby \\ Department of Mathematics, University of Oslo, Norway. E-mail: arne@math.uio.no
}

Erik Vanem

DNV-GL, Norway.E-mail: Erik.Vanem@dnvgl.com

\author{
Maria Hjelset Barbosa \\ Department of Mathematics, University of Oslo, Norway. E-mail: maria.hjelset@ gmail.com
}

Environmental contours are widely used as a basis for e.g., ship design, especially in early design phases. The traditional approach to such contours is based on the well-known Rosenblatt transformation. In the present paper we present a numerical method making it possible to apply the inverse Rosenblatt transformation to mixtures of distributions. Due to the effects of this transformation the probabilistic properties of the resulting environmental contour can be distorted. Based on a precise definition of the concept of exceedance probability, valid for all types of environmental contours, we show how to evaluate a given contour and adjust it so that it gets the desired properties. The methods are illustrated by a numerical example.

Keywords: Environmental contours, Rosenblatt transformation, mixtures, simulation.

\section{Introduction}

Environmental contours are widely used as a basis for e.g., ship design. Such contours are typically used in early design when the strength and failure properties of the object under consideration are not known. An environmental contour describes the tail properties of some relevant environmental variables, and is used as input to the design process. See Haver (1987), Baarholm et al. (2010), Ditlevsen (2002), Moan (2009) and Jonathan et al. (2011). The methodology for constructing environmental contours were introduced by Winterstein et al. (1993) and Haver and Winterstein (2009). The process starts out by constructing a contour for two independent standard normally distributed variables. This contour is then transformed to the environmental space using the inverse Rosenblatt transformation introduced in Rosenblatt (1952). As pointed out in Huseby et al. (2013) the probabilistic properties of the contour is typically not preserved under this transformation. Hence, the resulting contour may need to be adjusted in order to get the desired exceedance probability. Huseby et al. (2013) also presented an alternative approach where environmental contours are constructed using Monte Carlo simulation. For a similar approach to a related problem see Ottesen and Aarstein (2006). Improved methods are found in Huseby et al. (2015a) and Huseby et al. (2015b).

The Rosenblatt transformation and its inverse is defined for any absolutely continuous joint distri- bution of the environmental variables. Thus, the method introduced by Winterstein et al. (1993) is in principle applicable to any such joint distribution. In general, however, the transformation may need to be carried out using numerical methods. In the present paper we show how this can be done efficiently in cases where the joint distribution is a mixture of a finite number of distributions. Such mixtures occur in cases where the joint distribution depends on some background variable such as the season or the wind direction. An example of this can be found in Vanem and Huseby (2018). See also Winterstein (2016).

The Monte Carlo approach presented in Huseby et al. (2015b) uses importance sampling based on the inverse Rosenblatt transformation. Thus, the proposed numerical method for mixtures is relevant for this approach as well. In the present paper, however, we focus on the method introduced by Winterstein et al. (1993). As mentioned above such contours may need to be adjusted in order to get the desired exceedance probability. In the present paper we also provide a tight upper bound on this probability calculated using a new geometric method. Using this upper bound the necessary adjustments can be identified and carried out. We illustrate the proposed methods on a simple numerical example.

\section{Basic concepts}

In this paper we consider cases where the environmental conditions can be described by a vector

Proceedings of the 29th European Safety and Reliability Conference.

Edited by Michael Beer and Enrico Zio

Copyright (c) 2019 European Safety and Reliability Association.

Published by Research Publishing, Singapore.

ISBN: 978-981-11-2724-3; doi:10.3850/978-981-11-2724-3_0719-cd 
$(T, H) \in \mathbb{R}^{2}$. An environmental contour is then defined as the boundary of a set $\mathcal{B} \subseteq \mathbb{R}^{2}$, and denoted $\partial \mathcal{B}$.

A given mechanical structure can withstand environmental stress up to a certain level. The failure region of the structure is the set of states of the environmental variables that imply that the structure fails. The exact shape of the failure region of a structure may be unknown. Still it may be possible to argue that the failure region belongs to a certain family which we denote by $\mathcal{E}$. A given environmental contour $\partial \mathcal{B}$ will be evaluated with respect to this family. The family $\mathcal{E}$ is chosen relative to $\mathcal{B}$ such that for all failure regions $\mathcal{F} \in \mathcal{E}$ we have that $\mathcal{F} \cap \mathcal{B}_{o}=\emptyset$, where $\mathcal{B}_{o}=\mathcal{B} \backslash \partial \mathcal{B}$ denotes the interior of $\mathcal{B}$. Thus, a failure region $\mathcal{F}$ may intersect with the boundary of $\mathcal{B}$ but not the interior of $\mathcal{B}$.

The exceedence probability of $\mathcal{B}$ with respect to $\mathcal{E}$ was introduced in Huseby et al. (2017) and is defined as:

$$
P_{e}(\mathcal{B}, \mathcal{E})=\sup _{\mathcal{F} \in \mathcal{E}} P[(T, H) \in \mathcal{F}] .
$$

We observe that the exceedance probability defined above represents an upper bound on the failure probability of the structure assuming that the true failure region is a member of the family $\mathcal{E}$. Of particular interest are cases where one can argue that the failure region of a structure is convex. Throughout this paper we will assume that this is the case. Thus, for a given set $\mathcal{B}$ we let $\mathcal{E}$ be the class of all convex sets which do not intersect with $\mathcal{B}_{o}$.

\subsection{Transformed contours}

We now review the approach to environmental contours based on the well-known Rosenblatt transformation in the context of an exceedance probability defined relative to a family of failure regions. The Rosenblatt transformation, denoted $\Psi$, is such that if $(X, Y)=\Psi(T, H)$, then $X$ and $Y$ are independent standard normally distributed. See Winterstein et al. (1993).

A contour for the transformed vector $(X, Y)$ is constructed as follows: Let $p_{e}<0.5$ be the desired exceedance probability, and let $r>0$ denote the $\left(1-p_{e}\right)$-percentile in the standard normal distribution. We then let $\mathcal{B}^{\prime}$ be a circle centred at the origin and with radius $r$, and let $\mathcal{E}^{\prime}$ be the family of all convex sets $\mathcal{F}^{\prime}$ which do not intersect with $\mathcal{B}_{o}^{\prime}$. It is then easy to verify that:

$$
P_{e}\left(\mathcal{B}^{\prime}, \mathcal{E}^{\prime}\right)=\sup _{\mathcal{F}^{\prime} \in \mathcal{E}^{\prime}} P\left[(X, Y) \in \mathcal{F}^{\prime}\right]=p_{e} .
$$

Thus, the contour $\partial \mathcal{B}^{\prime}$ has the desired exceedance probability with respect to the family $\mathcal{E}^{\prime}$ of failure regions. For details see Huseby et al. (2017).
In order to obtain a contour set in the original space, the set $\mathcal{B}^{\prime}$ is transformed using the inverse Rosenblatt transformation, $\Psi^{-1}$, as follows:

$$
\mathcal{B}=\left\{(t, h)=\Psi^{-1}(x, y):(x, y) \in \mathcal{B}^{\prime}\right\} .
$$

Similarly, under mild regularity conditions the environmental contour $\partial \mathcal{B}$ is obtained from $\partial \mathcal{B}^{\prime}$ as:

$$
\partial \mathcal{B}=\left\{(t, h)=\Psi^{-1}(x, y):(x, y) \in \partial \mathcal{B}^{\prime}\right\} .
$$

In applied cases one has to simplify this transformation by transforming only a finite set of evenly spaced points, $\left(x_{1}, y_{1}\right), \ldots,\left(x_{m}, y_{m}\right) \in \partial \mathcal{B}^{\prime}$. We then let:

$$
\left(t_{i}, h_{i}\right)=\Psi^{-1}\left(x_{i}, y_{i}\right), \quad i=1, \ldots, m,
$$

and approximate $\partial \mathcal{B}$ by a polygon with the transformed points as corners. For convenience we also introduce the edges of this polygon, denoted $e_{1}, \ldots, e_{m}$, where $e_{i}$ is the line segment between $\left(t_{i}, h_{i}\right)$ and $\left(t_{i+1}, h_{i+1}\right), i=1, \ldots m-1$, and $e_{m}$ is the line segment between $\left(t_{m}, h_{m}\right)$ and $\left(t_{1}, h_{1}\right)$.

\section{Tail issues with the inverse Rosenblatt transformation}

As mentioned in the introduction the inverse Rosenblatt transformation can also be used as a very efficient tool in Monte Carlo simulation. See Huseby et al. (2015b) for details. However, when sampling from the tail of a distribution, there are some issues one needs to take into account. In this section we take a closer look at this, and assume that we are given a random vector $(X, Y)$ where $X$ and $Y$ are independent and standard normally distributed. The objective is to transform $(X, Y)$ to a vector $(T, H)$ with a given absolutely continuous joint distribution where:

$$
\begin{aligned}
& P(H \leq h)=F_{H}(h), \\
& \begin{aligned}
P(H & >h)=1-F_{H}(h) \\
& =\bar{F}_{H}(h), \\
P(T & \leq t \mid H=h)=F_{T \mid H}(t \mid h), \\
P(T & >t \mid H=h)=1-F_{T \mid H}(t \mid h) \\
& =\bar{F}_{T \mid H}(t \mid h) .
\end{aligned}
\end{aligned}
$$

This is done using the inverse Rosenblatt transformation which consists of two steps:

Step 1. Transform $(X, Y)$ to a vector $(U, V)$ where $U$ and $V$ are independent and uniformly distributed on $[0,1]$.

Step 2. Transform $(U, V)$ to a vector $(T, H)$ with the desired joint distribution. 
We denote the cumulative distribution function of the standard normal distribution by $\Phi$, and the survival function of the standard normal distribution by $\bar{\Phi}$.

In principle the first step of inverse Rosenblatt transformation can be carried out by transforming $(X, Y)$ to $(U, V)$ as follows:

$$
\begin{gathered}
U=\Phi(X), \\
V=\Phi(Y) .
\end{gathered}
$$

This implies that for all $u, v \in[0,1]$ we have:

$$
\begin{aligned}
P(U \leq u) & =P(\Phi(X) \leq u)=P\left(X \leq \Phi^{-1}(u)\right) \\
& =\Phi\left(\Phi^{-1}(u)\right)=u \\
P(V \leq v) & =P(\Phi(Y) \leq v)=P\left(Y \leq \Phi^{-1}(v)\right) \\
& =\Phi\left(\Phi^{-1}(v)\right)=v .
\end{aligned}
$$

Hence, $U$ and $V$ are independent and uniformly distributed on $[0,1]$.

The second step of the inverse Rosenblatt transformation where $(U, V)$ is transformed to $(T, H)$ is then done as follows:

$$
\begin{aligned}
& H=F_{H}^{-1}(U), \\
& T=F_{T \mid H}^{-1}(V \mid H) .
\end{aligned}
$$

This implies that for all $h, t$ we have:

$$
\begin{aligned}
P(H & \leq h)=P\left(F_{H}^{-1}(U) \leq h\right) \\
& =P\left(U \leq F_{H}(h)\right)=F_{H}(h), \\
P(T & \leq t \mid H=h)=P\left(F_{T \mid H}^{-1}(V \mid h) \leq t \mid H=h\right) \\
& =P\left(V \leq F_{T \mid H}(t \mid h) \mid H=h\right)=F_{T \mid H}(t \mid h) .
\end{aligned}
$$

When sampling from the upper tail of the normal distribution, however, this procedure can be numerically unstable. If $X$ and $Y$ are large positive numbers, the resulting values of $U$ and $V$ will be close to 1 . Such numbers will often be rounded up to 1 , and thus, yielding bad results in the second step of the transformation. In order to avoid these issues a modified procedure should be applied:

If $X \leq 0$, we let:

$$
\begin{aligned}
& U=\Phi(X), \\
& H=F_{H}^{-1}(U) .
\end{aligned}
$$

If $X>0$, we let:

$$
\begin{aligned}
& U=\bar{\Phi}(X), \\
& H=\bar{F}_{H}^{-1}(U) .
\end{aligned}
$$

If $Y \leq 0$, we let:

$$
\begin{aligned}
& V=\Phi(Y), \\
& T=F_{T \mid H}^{-1}(V \mid H) .
\end{aligned}
$$

If $Y>0$, we let:

$$
\begin{aligned}
& V=\bar{\Phi}(Y), \\
& T=\bar{F}_{T \mid H}^{-1}(V \mid H) .
\end{aligned}
$$

It is easy to verify that $(T, H)$ gets the correct joint distribution in this case as well. At the same time we keep $U$ and $V$ in the lower part of the interval $[0,1]$, and thus, we avoid the numerical issues with handling numbers close to the upper part of this interval.

\section{Mixtures of distributions}

We now consider a case where the joint distribution of $(T, H)$ is a mixture of distributions. In such cases it may not be possible to find explicit formulas for $F_{H}^{-1}$ and $F_{T \mid H}^{-1}$. Instead one has to find the inverse by solving an equation numerically. Here we explain how to do this for $F_{H}^{-1}$. The corresponding procedure for $F_{T \mid H}^{-1}$ is completely similar. More specifically, we assume that $F_{H, 1}, \ldots, F_{H, m}$ are $m$ cumulative distribution functions which are all continuous and strictly increasing. Moreover, we assume that the inverse functions $F_{H, 1}^{-1}, \ldots, F_{H, m}^{-1}$ are known and easy to calculate, and we introduce:

$$
h_{j}=F_{H, j}^{-1}(u), \quad j=1, \ldots, m .
$$

We also define:

$$
h_{\min }=\min _{1 \leq j \leq m} h_{j}, \text { and } h_{\max }=\max _{1 \leq j \leq m} h_{j} .
$$

We then introduce the cumulative distribution function for $H$ as the mixture of $F_{H, 1}, \ldots, F_{H, m}$ :

$$
F_{H}(h)=\sum_{j=1}^{m} \alpha_{j} F_{H, j}(h),
$$

where $\alpha_{j} \geq 0, j=1, \ldots, m$, and $\sum_{j=1}^{m} \alpha_{j}=1$.

We assume that we have found that $U=u$, and that we want to compute the corresponding value for $H, h=F_{H}^{-1}(u)$. This is equivalent to solving the following equation:

$$
F_{H}(h)=\sum_{j=1}^{m} \alpha_{j} F_{H, j}(h)=u .
$$

We then claim that if $h$ is the solution to (6), then:

$$
h_{\min } \leq h \leq h_{\max } .
$$


To prove (7) we note that since the cumulative distribution functions are non-decreasing and $\sum_{j=1}^{m} \alpha_{j}=1$, we have:

$$
\begin{aligned}
F_{H}\left(h_{m i n}\right) & =\sum_{j=1}^{m} \alpha_{j} F_{H, j}\left(h_{m i n}\right) \\
& \leq \sum_{j=1}^{m} \alpha_{j} F_{H, j}\left(h_{j}\right) \\
& =\sum_{j=1}^{m} \alpha_{j} u=u
\end{aligned}
$$

Similarly, we have:

$$
\begin{aligned}
F_{H}\left(h_{\max }\right) & =\sum_{j=1}^{m} \alpha_{j} F_{H, j}\left(h_{\max }\right) \\
& \geq \sum_{j=1}^{m} \alpha_{j} F_{H, j}\left(h_{j}\right) \\
& =\sum_{j=1}^{m} \alpha_{j} u=u
\end{aligned}
$$

Since $F_{H, 1}, \ldots, F_{H, m}$ are continuous and strictly increasing, it follows that $F_{H}$ is continuous and strictly increasing as well. Thus, since we have established that:

$$
F_{H}\left(h_{\min }\right) \leq u \leq F_{H}\left(h_{\max }\right)
$$

there must exist some $h \in\left[h_{\min }, h_{\max }\right]$ such that $F_{H}(h)=u$.

Having identified the interval $\left[h_{\min }, h_{\max }\right]$ which must contain the solution to (6), the solution can easily be found numerically, e.g., by using the bisection method.

\section{Exceedance probabilities of transformed contours}

The problem with transformed contours is that the exceedance probability is not preserved under this transformation in general. In fact, we typically have $P_{e}(\mathcal{B}, \mathcal{E})>p_{e}$. Thus, the contour may need to be inflated in order to get the correct exceedance probability. Before this can be done, however, we need to have a way of computing the exceedance probability of a given contour set $\mathcal{B}$.

If $\mathcal{B}$ is convex, the maximal failure regions will be half-spaces separated from $\mathcal{B}$ by tangents. Using Monte Carlo simulation the probability of having an outcome within such a half-space can be estimated. By running through all tangents along the border of $\mathcal{B}$, the failure region with the highest probability can be identified, and thus the exceedance probability can be estimated.
In a more general case, however, the set $\mathcal{B}$ may not be convex. In such cases it is difficult to run through all maximal failure regions in order to identify the one with the highest probability. Huseby et al. (2017) showed how to calculate an upper and a lower bound on the exceedance probability in such cases. In the present paper we suggest an improved upper bound. Comparisons with the lower bound show that this upper bound is in fact very close to the correct exceedance probability.

The main idea of this new approach is to work with a slightly modified family of failure regions. In order to explain this in detail we consider a contour set $\mathcal{B}$ and introduce:

$$
\tilde{\mathcal{E}}=\{\tilde{\mathcal{F}}(u): u \in \partial \mathcal{B}\},
$$

where $\tilde{\mathcal{F}}(u)$ is the set of all points $v \notin \mathcal{B}_{O}$ that are visible from $u$. A point $v \notin \mathcal{B}_{o}$ is said to be visible from $u$ if the line between $u$ and $v$ is not intersecting $\mathcal{B}_{o}$.

For an arbitrary failure region $\mathcal{F} \in \mathcal{E}$, there exists a maximal set $\mathcal{F}^{*} \in \mathcal{E}$ such that $\mathcal{F} \subseteq \mathcal{F}^{*}$ and such that $\mathcal{F}^{*}$ has at least one point, say $\bar{u}_{0}$, in common with the contour $\partial \mathcal{B}$, i.e., $u_{0} \in \mathcal{F}^{*} \cap \partial \mathcal{B}$. We now claim that $\mathcal{F}^{*} \subseteq \tilde{\mathcal{F}}\left(u_{0}\right)$.

In order to show this we need to show that all points in $\mathcal{F}^{*}$ are visible from $u_{0}$. From the definition of failure regions and contours we know that $\mathcal{F}^{*}$ does not intersect with $\mathcal{B}_{0}$. Since $\mathcal{F}^{*}$ is convex, we have that for any two points $u, v \in$ $\mathcal{F}^{*}$, every point on the line segment between $u$ and $v$ is also a member of $\mathcal{F}^{*}$. This means that all points in $\mathcal{F}^{*}$ are visible from all other points in the set. In particular, all points in $\mathcal{F}^{*}$ must be visible from $u_{0}$ implying that $\mathcal{F}^{*} \subseteq \tilde{\mathcal{F}}\left(u_{0}\right)$ as claimed. From this it also follows that $\mathcal{F} \subseteq \tilde{\mathcal{F}}\left(u_{0}\right)$.

Summarising this, we have shown that for each $\mathcal{F} \in \mathcal{E}$ there exists a set $\tilde{\mathcal{F}}\left(u_{0}\right) \in \tilde{\mathcal{E}}$ such that $\mathcal{F} \subseteq \tilde{\mathcal{F}}\left(u_{0}\right)$, and from this it follows that:

$$
P_{e}(\mathcal{B}, \mathcal{E}) \leq P_{e}(\mathcal{B}, \tilde{\mathcal{E}})
$$

Thus, $P_{e}(\mathcal{B}, \tilde{\mathcal{E}})$ is an upper bound on the exceedance probability.

It can be shown that if $\mathcal{B}$ is convex and $\partial \mathcal{B}$ is a differentiable curve, we get that:

$$
P_{e}(\mathcal{B}, \mathcal{E})=P_{e}(\mathcal{B}, \tilde{\mathcal{E}})
$$

Thus, in such cases $P_{e}(\mathcal{B}, \tilde{\mathcal{E}})$ is exact. Furthermore, in general the sets in $\tilde{\mathcal{E}}$ are almost convex. Thus, $P_{e}(\mathcal{B}, \tilde{\mathcal{E}})$ is typically a very good upper bound on the true exceedance probability.

\subsection{Estimating the upper bound}

In order to estimate the upper bound $P_{e}(\mathcal{B}, \tilde{\mathcal{E}})$ we recall that the set $\partial \mathcal{B}$ is approximated by a 
polygon with edges $e_{1}, \ldots, e_{m}$. We say that a point $v \notin \mathcal{B}_{O}$ is visible from the edge $e_{i}$ if it is visible from the midpoint of $e_{i}$, denoted $u_{i}$, $i=1, \ldots, m$. The family $\tilde{\mathcal{E}}$ is then approximated by the finite family:

$$
\tilde{\mathcal{E}}_{m}=\left\{\tilde{\mathcal{F}}\left(u_{i}\right): i=1, \ldots, m\right\},
$$

while the upper bound of the exceedance probability is approximated by:

$$
P_{e}\left(\mathcal{B}, \tilde{\mathcal{E}}_{m}\right)=\max _{1 \leq i \leq m} P\left[(T, H) \in \tilde{\mathcal{F}}\left(u_{i}\right)\right] .
$$

The $m$ probabilities on the right-hand side of the above formula can be estimated by Monte Carlo simulation. A simple method for doing this is to start out by generating $N$ points $\left(x_{1}, y_{1}\right), \ldots,\left(x_{N}, y_{N}\right)$ from the bivariate normal distribution. These points are then transformed using the inverse Rosenblatt transformation as explained in Sections 3 and 4. For simplicity, the transformed points are denoted by $v_{1}, \ldots, v_{N}$. The probability $P\left[(T, H) \in \tilde{\mathcal{F}}\left(u_{i}\right)\right]$ can then be estimated by:

$$
\hat{P}_{i}=\frac{1}{N} \sum_{j=1}^{N} I\left(v_{j} \in \tilde{\mathcal{F}}\left(u_{i}\right)\right), \quad i=1, \ldots, m .
$$

Note that this simulation method can be improved considerably by using importance sampling where we only sample points in the tail area of the bivariate normal distribution. A detailed description of how this can be done, however, is beyond the scope of the present paper. For more on this see Huseby et al. (2015b). This method is improved further in Barbosa (2018).

The remaining problem now is how to calculate the indicator functions:

$$
I\left(v_{j} \in \tilde{\mathcal{F}}\left(u_{i}\right)\right), \quad i=1, \ldots, m, \quad j=1, \ldots, N,
$$

in an efficient way. That is, for any given point $v_{j}$ we need to determine if this point is visible from $u_{i}, i=1, \ldots, m, j=1, \ldots, N$.

If the edge $e_{i}$ is a supporting hyperplane of $\mathcal{B}$, $v_{j}$ is visible from $u_{i}$ if and only if $v_{j}$ and $\mathcal{B}_{o}$ lie on opposite sides of $e_{i}$. This is very easy to check using standard geometric methods. If the edge $e_{i}$ is not a supporting hyperplane of $\mathcal{B}, v_{j}$ is visible from $u_{i}$ if and only if the line segment between $v_{j}$ and $u_{i}$ does not intersect with any of the other edges. An efficient way of checking this can be found in O'Rourke (1998).

Although both cases can be handled reasonably fast, the first procedure is by far the easiest one. In order to quickly distinguish between these two cases, it is convenient to identify all edges which are supporting hyperplanes before running the simulations. If this is done, the more complex procedure where $e_{i}$ is not a supporting hyperplane is easily avoided whenever possible.

Note that if $\mathcal{B}$ is convex, all edges are supporting hyperplanes of $\mathcal{B}$. Thus, such cases are very easy to handle.

\section{Numerical example}

In this subsection we illustrate the proposed method by considering a joint long-term model for significant wave height and wave period, denoted respectively by $H$ and $T$. The joint distribution for $H$ and $T$ is based on a model discussed in Vanem and Bitner-Gregersen (2015). In this model a marginal distribution is fitted to the data for significant wave height and a conditional model, conditioned on the value of significant wave height, is subsequently fitted to the wave period. The joint model has the following form:

$$
f_{T, H}(t, h)=f_{H}(h) f_{T \mid H}(t \mid h)
$$

Following Vanem and Bitner-Gregersen (2015) a three-parameter Weibull distribution is used for the significant wave height, $H$, and a lognormal conditional distribution is used for the wave period, $T$. The three-parameter Weibull distribution is parameterized by a location parameter, $\gamma$, a scale parameter $\alpha$, and a shape parameter $\beta$ as follows:

$$
f_{H}(h)=\frac{\beta}{\alpha}\left(\frac{h-\gamma}{\alpha}\right)^{\beta-1} e^{-[(h-\gamma) / \alpha]^{\beta}}, \quad h \geq \gamma .
$$

The lognormal distribution has two parameters, the log-mean $\mu$ and the log-standard deviation $\sigma$ and is expressed as:

$$
f_{T \mid H}(t \mid h)=\frac{1}{t \sqrt{2 \pi}} e^{-\left[(\ln (t)-\mu)^{2} /\left(2 \sigma^{2}\right)\right]}, \quad t \geq 0,
$$

where the dependence between $H$ and $T$ is modelled by letting the parameters $\mu$ and $\sigma$ be expressed in terms of $H$ as follows:

$$
\begin{gathered}
\mu=E[\ln (T) \mid H=h]=a_{1}+a_{2} h^{a_{3}}, \\
\sigma=S D[\ln (T) \mid H=h]=b_{1}+b_{2} e^{b_{3} h} .
\end{gathered}
$$

The parameters $a_{1}, a_{2}, a_{3}, b_{1}, b_{2}, b_{3}$ are typically estimated using available data from the relevant geographical location.

Vanem and Huseby (2018) consider a case where the joint model depends on the season. In order to handle this situation separate submodels are fitted for each season. The combined model is a mixture of all the submodels. As an illustration of the proposed method we consider a simple version of this with only five different submodels. Thus, the mixture model becomes:

$$
f_{T, H}(t, h)=\sum_{i=1}^{5} \lambda_{i} f_{H, i}(h) f_{T \mid H, i}(t \mid h),
$$


where the weights $\lambda_{1}, \ldots, \lambda_{5}$ are non-negative with sum 1, and where each submodel is of the same type as considered in Vanem and BitnerGregersen (2015).

The parameters for the three-parameter Weibull distributions are listed in Table 1, while the parameters for the conditional log-normal distributions are listed in Table 2. In a real life situation the parameter values are estimated based on relevant data. See Vanem and Huseby (2018). Here, however, the parameter values are just made-up examples chosen so that the different submodels represent a substantial range of conditions.

Table 1. Parameters for the five three-parameter Weibull distributions for significant wave heights.

\begin{tabular}{cccc}
\hline Submodel & $\alpha$ & $\beta$ & $\gamma$ \\
\hline 1 & 2.527 & 1.460 & 0.337 \\
2 & 2.517 & 1.470 & 0.327 \\
3 & 3.007 & 1.260 & 0.437 \\
4 & 2.007 & 1.560 & 0.299 \\
5 & 2.307 & 1.360 & 0.307 \\
\hline
\end{tabular}

Table 2. Parameters for the five conditional log-normal distributions for wave periods.

\begin{tabular}{ccccr}
\hline Submodel & & $i=1$ & $i=2$ & $i=3$ \\
\hline \multirow{2}{*}{1} & $a_{i}$ & 1.069 & 0.898 & 0.243 \\
& $b_{i}$ & 0.025 & 0.263 & -0.148 \\
2 & $a_{i}$ & 1.079 & 0.888 & 0.253 \\
& $b_{i}$ & 0.015 & 0.273 & -0.108 \\
3 & $a_{i}$ & 1.060 & 0.878 & 0.253 \\
& $b_{i}$ & 0.020 & 0.273 & -0.108 \\
4 & $a_{i}$ & 1.059 & 0.868 & 0.223 \\
& $b_{i}$ & 0.030 & 0.253 & -0.088 \\
5 & $a_{i}$ & 1.073 & 0.798 & 0.213 \\
& $b_{i}$ & 0.028 & 0.260 & -0.188 \\
\hline
\end{tabular}

We use a return period of 25 years, and assume that the models are fitted using sea states representing periods of 3 hours. Thus, we get 8 data points per 24 hours. The desired exceedance probability then becomes:

$$
P_{e}=\frac{1}{25 \cdot 365.25 \cdot 8}=1.37 \cdot 10^{-5} \text {. }
$$

For more details about how to calculate this we refer to Vanem and Bitner-Gregersen (2015).

Figure 1 shows the environmental contours for the five individual submodels before mixing. All curves are derived using the standard inverse Rosenblatt transformation. We note that the contours vary quite a lot. In particular the blue con- tour, representing Submodel 3, is much wider than the others.

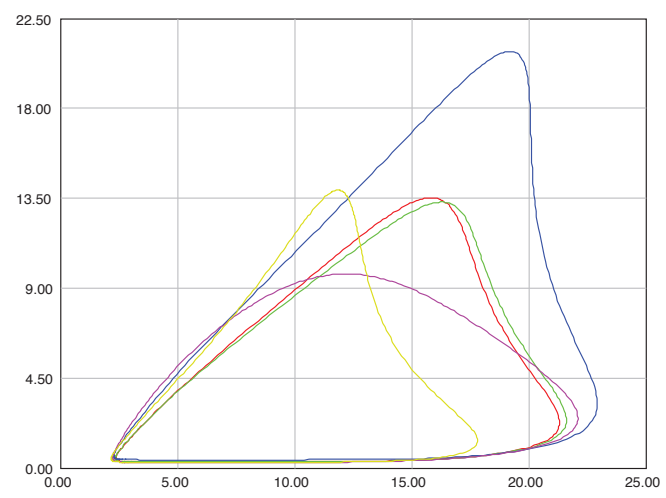

Fig. 1. Environmental contours for the five individual submodels before mixing. Submodel 1 (red curve), Submodel 2 (green curve), Submodel 3 (blue curve), Submodel 4 (pink curve) and Submodel 5 (yellow curve).

Figure 2 shows the resulting environmental contour for the mixture model. We observe that this contour to a certain extent inherits features from the submodel contours. Still the relation between this contour and the submodel contours is not trivial.

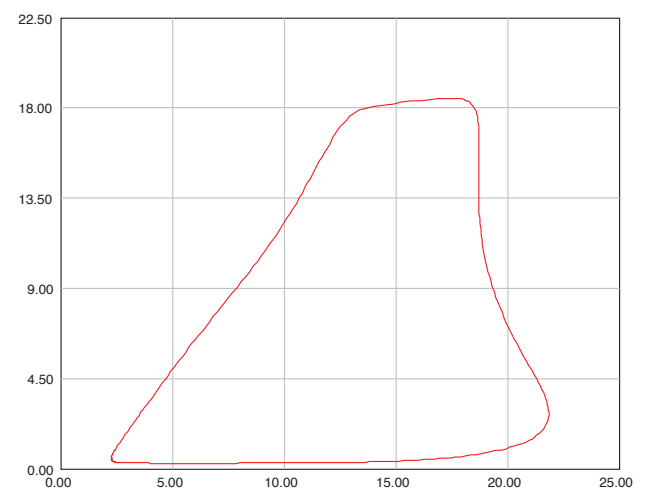

Fig. 2. Environmental contour for the mixed bivariate distribution.

By using Monte Carlo simulation and (8) we estimate probabilities for the sets $\tilde{\mathcal{F}}\left(u_{i}\right), i=$ $1, \ldots, m$ where $m=360$ as we let $u_{i}$ run along the contour. The resulting probabilities are represented by the green curve in Figure 3 . The desired exceedance probability, $1.37 \cdot 10^{-5}$ is shown as the red curve in the same diagram. We see that 
the probabilities for the sets vary a lot. In some areas the green curve is way below the red curve. At the peaks, however, the estimated probabilities are much larger than the desired probability. More specifically, the estimated probability for $\tilde{\mathcal{F}}\left(u_{105}\right)$ is $3.74 \cdot 10^{-5}$, while the corresponding number for $\tilde{\mathcal{F}}\left(u_{205}\right)$ is $4.19 \cdot 10^{-5}$. In Figure 4 we have shown the simulated outcomes for $\tilde{\mathcal{F}}\left(u_{105}\right)$ and $\tilde{\mathcal{F}}\left(u_{205}\right)$ along with the environmental contour.

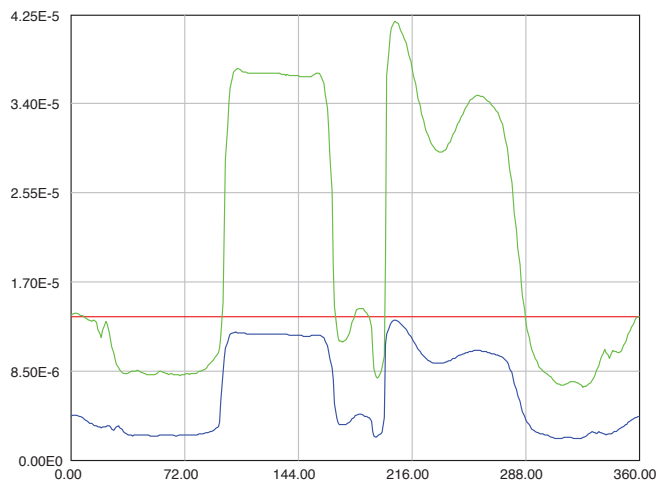

Fig. 3. Estimated probabilities for the sets $\tilde{\mathcal{F}}\left(u_{i}\right), i=$ $1, \ldots, 360$ for the original environmental contour (green curve) and the adjusted environmental contour (blue curve). Desired exceedance probability (red curve).

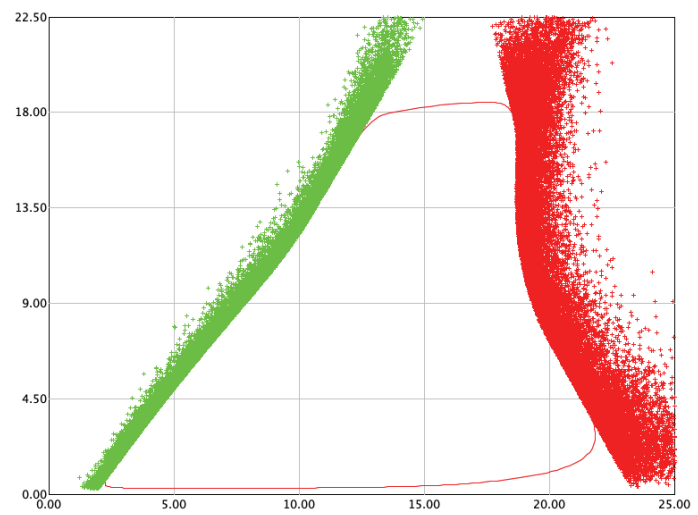

Fig. 4. Environmental contour along with simulated outcomes in the sets $\tilde{\mathcal{F}}\left(u_{105}\right)$ (red scatter) and $\tilde{\mathcal{F}}\left(u_{205}\right)$ (green scatter).

In order to adjust the environmental contour so that it gets the desired exceedance probability, we multiply the desired return period of 25 years by a factor and calculate the resulting exceedance probability. Using an iterative procedure this factor is adjusted until the desired exceedance probability is reached. The correct factor for this particular case turns out to be 3.28. Thus, when the environmental contour is constructed using the inverse Rosenblatt transformation, we need to use a return period of $3.28 \cdot 25=82$ years in order to get the desired exceedance probability.

In Figure 3 we have shown the resulting probabilities (the blue curve) along with the original probabilities (the green curve). We see that the blue curve is below the red curve all the way. The resulting adjusted environmental contour is shown in Figure 5 (green curve) along with the original contour (red curve). We observe that the adjusted environmental contour is clearly larger than the original contour. At the same time the blue curve in Figure 3 is mostly way below the red curve representing the desired probability. This indicates that the adjusted environmental contour may be too conservative in most areas. This, however, is a price one has to pay when using the inverse Rosenblatt transformation.

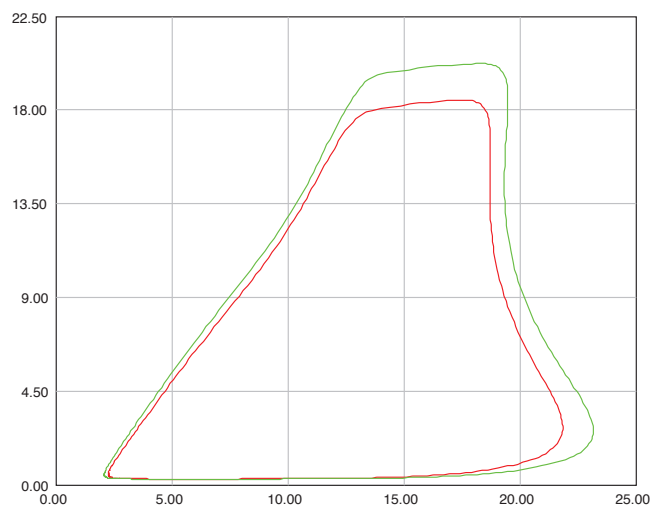

Fig. 5. Original (red curve) and adjusted (green curve) environmental contours for the mixed bivariate distribution.

\section{Conclusions}

In the present paper we have presented a numerical method making it possible to apply the inverse Rosenblatt transformation to mixtures of distributions. Moreover, we have shown how the probabilistic properties of the resulting environmental contour can be evaluated using a tight upper bound on the exceedance probability. This upper bound can be computed very efficiently using a combination of importance sampling and geometrical methods. For convex contours the upper bound is equal to the exact exceedance probability. Moreover, the convexity can be utilised in order to make the calculations even more efficient.

In the paper we also discuss some numerical issues related to sampling from the tail area. Han- 
dling these issues in a numerically stable way is very important in relation to importance sampling. Thus, the proposed solutions are relevant when a given contour is evaluated as well as in the process of constructing a contour using Monte Carlo simulation as suggested in Huseby et al. (2015a) and Huseby et al. (2015b).

The proposed methods are illustrated by considering an example where the joint distribution of the environmental variables is a mixture of five individual distributions. We also show how an environmental contour can be adjusted to get the desired exceedance probability by using an iterative algorithm. As a result of this adjustment, however, the contour becomes very conservative except at a few points along the border. Thus, in real-life applications such adjustments should be combined with an assessment of which parts of the contour that are critical for the given structure. More specifically, one should consider the sets $\tilde{\mathcal{F}}\left(u_{i}\right)$, and assess how likely it is that the true failure region of a given structure matches any of these. Alternatively, one could construct an adjusted contour where the adjustments are done only in certain critical areas.

\section{Acknowledgements}

This paper has been written with support from the Research Council of Norway (RCN) through the project ECSADES Environmental Contours for Safe Design of Ships and other marine structures.

\section{References}

Baarholm, G. S., S. Haver, and O. D. Økland (2010). Combining contours of significant wave height and peak period with platform response distributions for predicting design response. Marine Structures 23, 147-163.

Barbosa, M. H. (2018). Improved Method for Calculating Exceedance Probabilities of Environmental Contours. Master's Thesis, University of Oslo.

Ditlevsen, O. (2002). Stochastic model for joint wave and wind loads on offshore structures. Structural Safety 24, 139-163.

Haver, S. (1987). On the joint distribution of heights and periods of sea waves. Ocean Engineering 14, 359-376.

Haver, S. and S. R. Winterstein (2009). Environmental contour lines: A method for estimating long term extremes by a short term analysis. Transactions of the Society of Naval Architects and Marine Engineers 116, 116-127.

Huseby, A. B., E. Vanem, and K. Eskeland (2017). Evaluating properties of environmental contours. In M. Cepin and R. Bris (Eds.), Safety and Reliability, Theory and Applications. Proceedings of the Euro- pean safety and reliability Conference, pp. 21012109. CRC Press.

Huseby, A. B., E. Vanem, and B. Natvig (2013). A new approach to environmental contours for ocean engineering applications based on direct Monte Carlo simulations. Ocean Engineering 60, 124-135.

Huseby, A. B., E. Vanem, and B. Natvig (2015a). Alternative environmental contours for structural reliability analysis. Structural Safety 54, 32-45.

Huseby, A. B., E. Vanem, and B. Natvig (2015b). A new Monte Carlo method for environmental contour estimation. In T. Nowakowski, M. Młyńczak, A. Jodejko-Pietruczuk, and S. Werbińska-Wojciechowska (Eds.), Safety and Reliability: Methodology and Applications, Proceedings of the European safety and reliability Conference, pp. 2091-2098. Taylor \& Francis.

Jonathan, P., K. Ewans, and J. Flynn (2011). On the estimation of ocean engineering design contours. In Proc. 30th International Conference on Offshore Mechanics and Arctic Engineering, pp. 1-8.

Moan, T. (2009). Development of accidental collapse limit state criteria for offshore structures. Structural Safety 31, 124-135.

O'Rourke, J. (1998). Computational geometry in C. Cambridge university press.

Ottesen, T. and J. A. Aarstein (2006). The statistical boundary polygon of a two parameter stochastic process. In Proc. 25th International Conference on Offshore Mechanics and Arctic Engineering, pp. 16.

Rosenblatt, M. (1952). Remarks on a multivariate transformation. The Annals of Mathematical Statistics 23, 470-472.

Vanem, E. and E. Bitner-Gregersen (2015). Alternative environmental contours for marine structural design - a comparison study. Journal of Offshore Mechanics and Arctic Engineering 137: 051601-1-051601-8.

Vanem, E. and A. B. Huseby (2018). Seasonal and omni-seasonal environmental contours for extreme sea states. In Proc. 7th International Maritime Conference on Design for Safety, DfS 2018. Kobe, Japan: Osaka University, pp. 470-477.

Winterstein, S. R. (2016). Environmental Contours for sub-populations: Effects of wind-wave direction and cut-out wind speed. Technical Report TN 2016-071, Probability-Based Engineering.

Winterstein, S. R., T. C. Ude, C. A. Cornell, P. Bjerager, and S. Haver (1993). Environmental parameters for extreme response: Inverse form with omission factors. In Proc. 6th International Conference on Structural Safety and Reliability, pp. 551-557. 